\title{
Diagnóstico de sospecha del cáncer en los servicios de urgencia hospitalarios
}

\section{Suspected diagnosis of cancer in hospital emergency services}

\author{
A. Lana ${ }^{1,2}$, S. Álvarez-Guerrero ${ }^{3}$, P. Herrero-Puente ${ }^{3}$, M.V. Folgueras ${ }^{2,3}$, M.L. López ${ }^{1,2}$
}

\section{RESUMEN}

Fundamento. Medir la frecuencia, tendencia y distribución de los cánceres con diagnóstico de sospecha en los Servicios de Urgencia Hospitalarios (SUH) de Asturias durante 2006-12. Caracterizar clínicamente dichos cánceres y determinar si se diferencian de aquellos cuyo diagnóstico es realizado en otros servicios.

Métodos. Estudio descriptivo poblacional de los cánceres del Registro Hospitalario de Tumores de Asturias (España), que recoge características del paciente, del tumor (localización, histología, estadio, metástasis y demora), el hospital y el servicio que diagnostica. Se seleccionaron los pacientes con diagnóstico confirmado de cáncer (excepto piel no melanoma) en el periodo de estudio $(\mathrm{N}=26.020)$. Se buscaron diferencias entre los casos según el servicio que había realizado la sospecha diagnóstica. Se ejecutó un análisis de regresión del tiempo transcurrido entre el primer síntoma y el diagnóstico de sospecha, el diagnóstico definitivo y el tratamiento, controlando los principales confusores.

Resultados. El 7,9\% (n=2.056) de todos los cánceres se sospechó en un SUH (mínimo anual 5,3 y máximo 10,4, con tendencia creciente). Estos pacientes fueron principalmente varones (60,6\%), con edad media de 67,7 años, y cáncer en pulmón $(21,0 \%)$ y colon $(15,5 \%)$. Los SUH ocuparon el $6^{\circ}$ puesto en el ranking de servicios que diagnosticaron tumores. En los SUH hubo más diagnóstico de sospecha de tumores avanzados (33,0\%) y más metástasis $(29,5 \%)$. Los SUH redujeron a la mitad los tiempos entre el primer síntoma y DS (-64,3 días), y entre diagnóstico definitivo y el inicio del tratamiento (-15,9 días) con respecto al resto de servicios.

Conclusiones. Los SUH contribuyen de forma importante al diagnóstico de sospecha del cáncer, principalmente avanzado y metastásico, en sistema respiratorio y digestivo, cuyos síntomas escapan al diagnóstico accidental realizado en atención primaria, y debutan abruptamente.

Palabras clave. Servicio de urgencias. Cáncer. Diagnóstico. Investigación de servicio de salud. Estudios descriptivos.

\begin{abstract}
Background. To measure the frequency, trends and distribution of cancers with suspected diagnosis in the Hospital Emergency Services (HES) in Asturias during 2006-12. To describe the clinical characteristics of these cancers and to determine if they differ from those whose diagnosis is made in other services.
\end{abstract}

Methods. Population based descriptive study of cancers registered in the Hospital Tumour Registry of Asturias (Spain), which provided data of patient characteristics, cancer variables (site, histology, stage, metastasis and delay), the hospital and service of diagnosis. Patients with confirmed diagnosis of cancer (non-melanoma of skin excluded) in the study period were included $(\mathrm{N}=26,020)$. Differences of cancer cases according to the service that had performed the suspected diagnosis were analyzed. We performed regression analysis of the time between the first symptom and the suspected diagnosis, the definitive diagnosis and treatment, controlling main confounders.

Results. Seven point nine percent $(n=2,056)$ of all cancer cases were suspected in a HES (annual minimum of $5.3 \%$ and maximum $10.4 \%$, with an upward trend). These patients were mainly men $(60.6 \%)$, with a mean age of 67.7 years, and with lung $(21.0 \%)$ and colon cancer (15.5\%). The HES ranks 6 th place in the list of services which diagnosed cancer. There was more diagnosis of advanced tumours (33.0\%) and metastasis (29.5\%) in the HES. The HES halved the time between the first symptom and the SD ( -64.3 days; $\mathrm{p}<0.001)$, and between definitive diagnosis and initiation of treatment $(-15.9$ days; $\mathrm{p}<0.001)$ compared to the other services.

Conclusions. The HES contribute significantly to suspected cancer diagnosis, mainly advanced and metastatic tumours in the respiratory and digestive system, whose symptoms escape accidental diagnosis conducted in primary care, and they start abruptly.

Key words. Emergency Service. Neoplasms. Diagnosis. Health Services Research. Cross-sectional studies.
1. Facultad de Medicina y Ciencias de la Salud. Departamento de Medicina. Oviedo.

2. Instituto Universitario de Oncología del Principado de Asturias. Oviedo.

3. Hospital Universitario Central de Asturias. Oviedo.

Recepción: 12 de septiembre de 2013

Aceptación provisional: 15 de octubre de 2013

Aceptación definitiva: 19 de diciembre de 2013

\section{Correspondencia:}

Alberto Lana

Facultad de Medicina y Ciencias de la Salud Departamento de Medicina, $7^{\circ}$ planta dcha. Despacho 5

Avda. Julián Clavería, s/n

33006 Oviedo

E-mail. lanaalberto@uniovi.es 


\section{INTRODUCCIÓN}

La magnitud y trascendencia del cáncer es enorme. En la Unión Europea se diagnostican anualmente más de 2,4 millones de casos, el 19,3\% de todos los casos mundiales $^{1}$. España tiene tasas de incidencias por debajo de la media ${ }^{2}$; no obstante, se estima que entre 2006 y 2012 el número de casos se incrementó un $11 \%$, fundamentalmente debido al aumento del número de casos en mujeres ${ }^{3}$. Colon, pulmón, próstata y mama fueron, en ese orden, las localizaciones más frecuentes en los registros españoles y suponen 4 de cada $10 \operatorname{casos}^{1}$. En lo que se refiere a la mortalidad, Bray y col$^{4}$ prevén que el número de defunciones se incremente un $75 \%$ en 2030. En España, el número de fallecidos por tumores malignos se incrementó un $12,8 \%$ en la primera década del siglo $\mathrm{XXI}^{5} \mathrm{y}$, aunque cuestiones demográficas explican gran parte de este aumento, lo cierto es que este dato subraya la trascendencia creciente de esta enfermedad. Consecuentemente, se ha incrementado la discapacidad que genera el cáncer, que ya supone el $16 \%$ de la carga total de enfermedad de la población española ${ }^{6}$.

El término "urgencia oncológica" hace referencia a aquella que requiere asistencia rápida por síntomas relacionados con un cáncer establecido o su tratamiento ${ }^{7}$ y están aumentando por el incremento de la prevalencia y la supervivencia del cáncer en los países desarrollados ${ }^{8}$. Existe otro tipo de urgencias relacionadas con la oncología que afecta a pacientes antes de que se haya establecido el diagnóstico y cuyo paso por los servicios de urgencias hospitalarias (SUH) da lugar al diagnóstico de sospecha (DS) de cáncer. Parece que el DS se realiza más habitualmente en Atención Primaria (AP) ${ }^{9,10}$; pero, en ocasiones, sea por exacerbación o agravamiento de síntomas inespecíficos que no han alertado al facultativo de AP, sea porque el cáncer debuta como una urgencia, el Servicio de Urgencias Hospitalario (SUH) es el lugar al que el paciente acude ${ }^{7,11}$.

Existen muchos estudios sobre atención urgente y paciente oncológico. La mayoría se centra en describir los síntomas por los que el paciente requiere asistencia cuando el cáncer ya está diagnosticado ${ }^{12}$ o el tratamiento que recibe por una complicación concreta ${ }^{13-15}$. Sin embargo, no se conoce qué porcentaje del DS de cáncer se hace en el SUH y cuáles son las características epidemiológicas de los pacientes en los que el diagnóstico se inicia de esa manera. Los objetivos de nuestro estudio fueron: 1) medir la frecuencia de los cánceres confirmados en 2006-12 cuya sospecha diagnóstica fue realizada en todos los SUH de Asturias, su tendencia y su distribución hospitalaria; y 2) caracterizar el perfil clínico y epidemiológico de dichos cánceres y determinar si se diferencia significativamente de aquellos cuya presunción diagnóstica es realizada en otros servicios.

\section{MATERIAL Y MÉTODOS}

\section{Diseño del estudio y participantes}

Estudio descriptivo, longitudinal y retrospectivo de los pacientes con cáncer, cuyo diagnóstico de confirmación se realizó entre el 1 de enero de 2006 y el 31 de diciembre de 2012 en todos los centros asistenciales de Asturias (1.077.360 de habitantes en 2012). En el 96,8\% de los casos, la confirmación diagnóstica fue emitida por el servicio de Anatomía Patológica, aunque en un pequeño porcentaje el diagnóstico fue realizado por otros métodos, fundamentalmente clínicos o radiológicos. Se excluyeron aquellos pacientes con cánceres de piel no melanoma.

Desde el año 2004, el Registro Hospitalario de Tumores del Servicio de Salud de Principado de Asturias (RHT-SESPA), recopila los datos de todos los casos diagnosticados o tratados en los centros hospitalarios asturianos, públicos o privados, que pertenezcan a la Red Nacional de RHT $^{16}$. Este registro proporciona una base de datos anónima, con información clínica y epidemiológica básica de los pacientes referidos. De los 37.340 casos de cáncer en Asturias en el septenio 2006-2012, se excluyeron 11.320 casos que no tenían información del servicio que realizó el DS. Por tanto, los análisis se realizaron sobre 26.020 diagnósticos de cáncer. 
Se obtuvo el consentimiento del Comité Científico del RHT-SESPA y la aprobación del Comité Ético de Investigación Clínica del Principado de Asturias.

\section{Variables del estudio}

El RHT-SESPA dispone de información del servicio en el que se realizó el DS de cáncer, es decir, aquella unidad o nivel asistencial (hospital o centro de AP) en la que los profesionales médicos, ante los signos y síntomas del paciente, dejaron constancia por primera vez en la historia clínica de la presunción de un tumor. Entre estos servicios está incluido el SUH de todos los centros asistenciales de Asturias. Asimismo, se obtuvieron datos relacionados con el paciente (sexo y edad al diagnóstico), el cáncer (localización topográfica del tumor (categoría: según CIE-O-3 reagrupadas posteriormente en órganos y sistemas) ${ }^{17}$; estadio (desconocido, local, regional, avanzado, no aplicable); histología (en base a subtipos de CIE-O-3) ${ }^{17}$; presencia de metástasis y cáncer múltiple) y los tiempos entre (a) el primer síntoma y el DS; (b) el DS y la confirmación diagnóstica; y (c) el diagnóstico definitivo y el inicio del tratamiento. Como fecha de primer síntoma se tiene en cuenta aquella en la que el paciente refiere por primera vez alteraciones relacionadas con el tumor y como fecha del DS el día que el clínico sospecha su existencia por primera vez.

\section{Análisis de los datos}

La información proporcionada se transformó en una base de datos SPSS v.19.0 (SPSS Inc., Chicago, Illinois) para su análisis estadístico. Se realizó una depuración y control de calidad de los datos. Las variables cuantitativas se expresaron como medias y desviaciones estándar $(\sigma)$ y las cualitativas como frecuencias absolutas y porcentajes. Para contrastar las diferencias entre los cánceres con DS en los SUH y los de otros servicios agrupados, se realizaron pruebas bivariantes: prueba $\mathrm{Z}$ con la corrección de Bonferroni y prueba $\chi^{2}$ de Pearson, para la comparación de proporciones y prueba t de Student para la comparación de medias. Se realizó un análisis multivariante mediante regresión lineal múltiple para controlar el efecto de las potenciales variables confusoras (edad, sexo, estadio y metástasis) sobre el tiempo transcurrido entre los primeros síntomas y el DS, entre el DS y el diagnóstico de confirmación, y entre este y el inicio del tratamiento.

\section{RESULTADOS}

Durante el periodo estudiado, en los SUH se diagnosticaron presuntivamente 2.056 casos $(7,9 \%)$ del total de casos de cáncer $(\mathrm{N}=37.340)$, con un mínimo en 2008 del $5,3 \%$ y con una tendencia creciente hasta alcanzar el máximo en 2012 (10,4\%) (Fig. 1). Los SUH ocuparon el $6^{\circ}$ puesto en el ranking de servicios con mayor frecuencia de DS de cáncer, por detrás de Urología (15,5\%), Ginecología (13,1\%), Digestivo (11,1\%), Cirugía general y digestiva $(10,7 \%)$ y Medicina interna $(8,8 \%)$, y por delante de $\mathrm{AP}(6,8 \%)$. La edad media de los pacientes con DS en los SUH fue 67,7 años $(\sigma=15,2)$, significativamente superior a la encontrada en el resto de servicios (65,6 años; $\sigma=14,7 ; \mathrm{p}<0,001)$. El $60,6 \%$ de los pacientes con DS en el SUH eran hombres, porcentaje superior al del resto de servicios $(55,2 \% ; \mathrm{p}<0,001)$.

La tabla 1 muestra la localización y las principales características de los tumores más frecuentes en función del servicio que realizó el DS de cáncer, y las diferencias entre el SUH y los demás. El cáncer de pulmón y tráquea es el más sospechado en los SUH (21,0\%), mientras que en el resto de servicios fue el de mama (12,9\%). Aproximadamente 1 de cada 3 cánceres con DS en los SUH tenía un estadio avanzado ( $33,0 \%$ ), mientras que en el resto de servicios esta circunstancia fue menos habitual (14,9\%). En cuanto a la histología, la mayor parte de los tumores fue de tipo "glandular" y las diferencias entre servicios mostraron que en los SUH había, comparado con el resto de servicios, una mayor frecuencia de "otros epiteliales", "sistema nervioso", "sarcomas" y "mal definidos". En la tabla 2 se puede observar la distribución de las localizaciones según servicio de sospecha, esta vez agrupadas para simplificar la potencial toma de decisiones. 


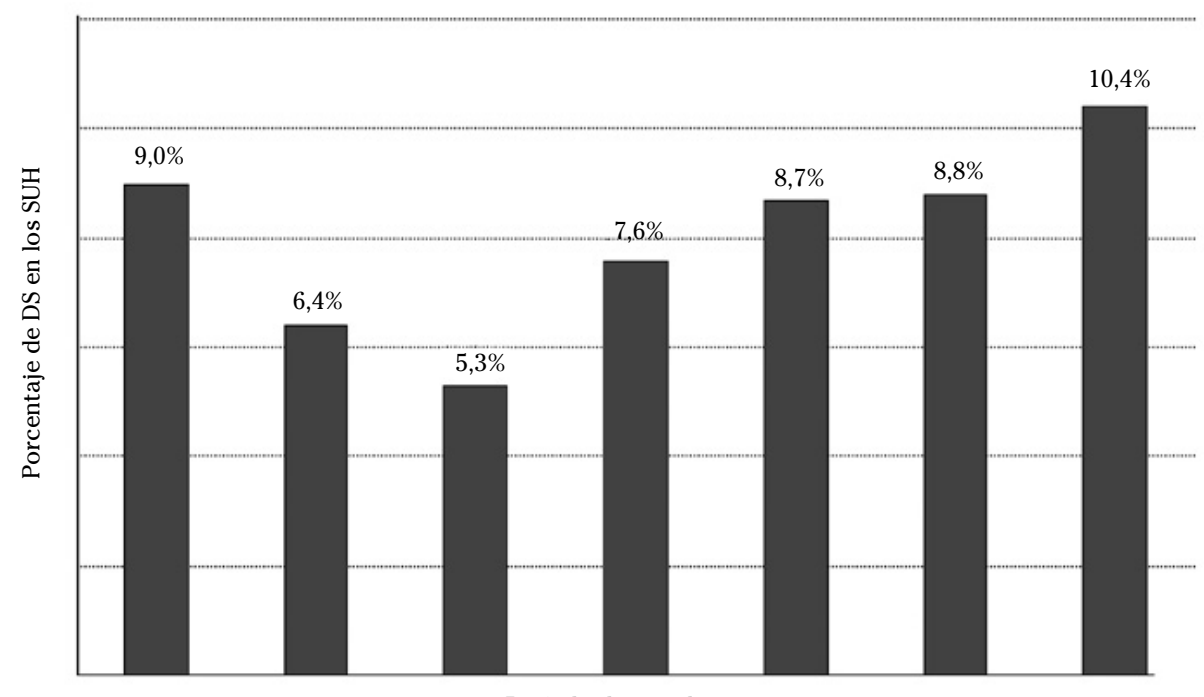

Período de estudio

Figura 1. Evolución porcentual de los diagnósticos de sospecha en los SUH por año. Asturias 2006-12.

Tabla 1. Características de localización, estadio e histología de los cánceres según el servicio de sospecha diagnóstica: SUH vs. otros servicios agrupados.

\begin{tabular}{lccccc}
\hline & \multicolumn{2}{c}{ Urgencias } & \multicolumn{2}{c}{ Otros servicios } & \multirow{2}{*}{ p-valor } \\
\cline { 2 - 5 } Localización & $\mathbf{n}$ & $\mathbf{\%}$ & $\mathbf{n}$ & $\mathbf{\%}$ & \\
\hline Tráquea y pulmón & & & & & \\
\hline Colon & 431 & 21,0 & 2.289 & 9,6 & $<0,001$ \\
\hline Estómago & 319 & 15,5 & 2.761 & 11,5 & $<0,001$ \\
\hline Encéfalo & 135 & 6,6 & 881 & 3,7 & $<0,001$ \\
\hline Vejiga urinaria & 120 & 5,8 & 180 & 0,8 & $<0,001$ \\
\hline Primario desconocido & 117 & 5,7 & 1.629 & 6,8 & 0,056 \\
\hline Hematopoyético & 103 & 5,0 & 511 & 2,1 & $<0,001$ \\
\hline Páncreas & 101 & 4,9 & 1.338 & 5,6 & 0,210 \\
\hline Mama & 82 & 4,0 & 403 & 1,7 & $<0,001$ \\
\hline Recto-ano & 81 & 3,9 & 3.092 & 12,9 & $<0,001$ \\
\hline Riñón & 78 & 3,8 & 915 & 3,8 & 0,994 \\
\hline Ovario & 62 & 3,0 & 603 & 2,5 & 0,165 \\
\hline Esófago & 53 & 2,6 & 375 & 1,6 & 0,002 \\
\hline Linfoide & 41 & 2,0 & 228 & 1,0 & $<0,001$ \\
\hline Hígado & 36 & 1,8 & 561 & 2,3 & 0,095 \\
\hline Cuerpo uterino & 32 & 1,6 & 380 & 1,6 & 0,990 \\
\hline Próstata & 30 & 1,5 & 695 & 2,9 & $<0,001$ \\
\hline Vesícula y vías biliares & 30 & 1,5 & 2.456 & 10,2 & $<0,001$ \\
\hline Meninges & 20 & 1,0 & 133 & 0,6 & 0,023 \\
\hline Cuello uterino & 20 & 1,0 & 98 & 0,4 & $<0,001$ \\
\hline Resto de localizaciones & 17 & 0,8 & 1.106 & 4,6 & $<0,001$ \\
\hline & 148 & 7,2 & 3.330 & 13,9 & $<0,001$ \\
\hline & & & & &
\end{tabular}




\begin{tabular}{|c|c|c|c|c|c|}
\hline & \multicolumn{2}{|c|}{ Urgencias } & \multicolumn{2}{|c|}{ Otros servicios } & \multirow{2}{*}{ p-valor } \\
\hline & $\mathbf{n}$ & $\%$ & $\mathbf{n}$ & $\%$ & \\
\hline \multicolumn{6}{|l|}{ Estadio } \\
\hline Local & 584 & 28,4 & 10.774 & 45,0 & $<0,001$ \\
\hline Regional & 341 & 16,6 & 3.787 & 15,8 & 0,346 \\
\hline Avanzado & 678 & 33,0 & 3.565 & 14,9 & $<0,001$ \\
\hline Desconocido & 66 & 3,2 & 1.455 & 6,1 & $<0,001$ \\
\hline No aplicable & 387 & 18,8 & 4.383 & 18,3 & 0,554 \\
\hline \multicolumn{6}{|l|}{ Histología } \\
\hline Escamoso y basocelular & 212 & 10,3 & 3.583 & 15,0 & $<0,001$ \\
\hline Glandular & 1057 & 51,4 & 13.495 & 56,3 & $<0,001$ \\
\hline Otros epiteliales & 202 & 9,8 & 1.158 & 4,8 & 0,031 \\
\hline Transicional & 118 & 5,7 & 1.680 & 7,0 & $<0,001$ \\
\hline Sistema nervioso & 131 & 6,4 & 312 & 1,3 & $<0,001$ \\
\hline Melanoma & 5 & 0,2 & 640 & 2,7 & $<0,001$ \\
\hline Sarcoma & 44 & 2,1 & 359 & 1,5 & 0,028 \\
\hline Linfoma & 62 & 3,0 & 853 & 3,6 & 0,214 \\
\hline Eritropoyético & 99 & 4,8 & 1.305 & 5,4 & 0,173 \\
\hline Gonadal y germinal & 14 & 0,7 & 110 & 0,5 & 0,178 \\
\hline Mixto & 6 & 0,3 & 54 & 0,2 & 0,474 \\
\hline Mal definido & 106 & 5,2 & 415 & 1,7 & $<0,001$ \\
\hline
\end{tabular}

Tabla 2. Localización agrupada más frecuente del cáncer según el servicio de sospecha diagnóstica.

\begin{tabular}{lccccc}
\hline & \multicolumn{2}{c}{ Urgencias } & \multicolumn{2}{c}{ Otros servicios } & p-valor \\
\cline { 2 - 5 } & $\mathbf{n}$ & $\%$ & $\mathbf{n}$ & $\%$ & \\
\hline Sistema digestivo & 733 & 35,7 & 5.892 & 24,6 & $<0,001$ \\
\hline Sistema respiratorio y órganos intratorácicos & 437 & 21,3 & 2.362 & 9,9 & $<0,001$ \\
\hline Sistema urinario & 185 & 9,0 & 2.322 & 9,7 & 0,247 \\
\hline Sistema nervioso & 145 & 7,1 & 348 & 1,5 & $<0,001$ \\
\hline Sistema hematopoyético y linfático & 137 & 6,7 & 1.899 & 7,9 & 0,042 \\
\hline Órganos genitales femeninos & 106 & 5,2 & 2.357 & 9,8 & $<0,001$ \\
\hline Primario desconocido & 103 & 5,0 & 511 & 2,1 & 0,004 \\
\hline Mama & 81 & 3,9 & 3.092 & 12,9 & $<0,001$ \\
\hline Órganos de cabeza y cuello & 56 & 2,7 & 1.502 & 6,3 & $<0,001$ \\
\hline Órganos genitales masculinos & 48 & 2,3 & 2.618 & 10,9 & $<0,001$ \\
\hline Huesos, cartílagos, articulaciones y tejidos blandos & 13 & 0,6 & 180 & 0,8 & 0,426 \\
\hline Sistema endocrino & 5 & 0,2 & 244 & 1,0 & 0,021 \\
\hline Loc ${ }^{0}$ alización mal definida & 5 & 0,2 & 31 & 0,1 & 0,258 \\
\hline Piel, melanoma & 2 & 0,1 & 606 & 2,5 & $<0,001$ \\
\hline
\end{tabular}


En el 29,5\% de los tumores con DS en los SUH se detectó una o más metástasis, porcentaje significativamente superior al del resto de servicios $(13,0 \% ; \mathrm{p}<0,001)$. Los cánceres múltiples fueron menos frecuentes en los SUH: un $15,0 \%$ frente al $19,1 \%$ en el resto de servicios $(\mathrm{p}<0,001)$.
La media de días de los diferentes periodos de tiempo estudiados es significativamente inferior cuando el cáncer es sospechado en los SUH (Fig. 2). Además, estas diferencias se mantienen cuando se controla la edad, el sexo, el estadio y la presencia de metástasis.

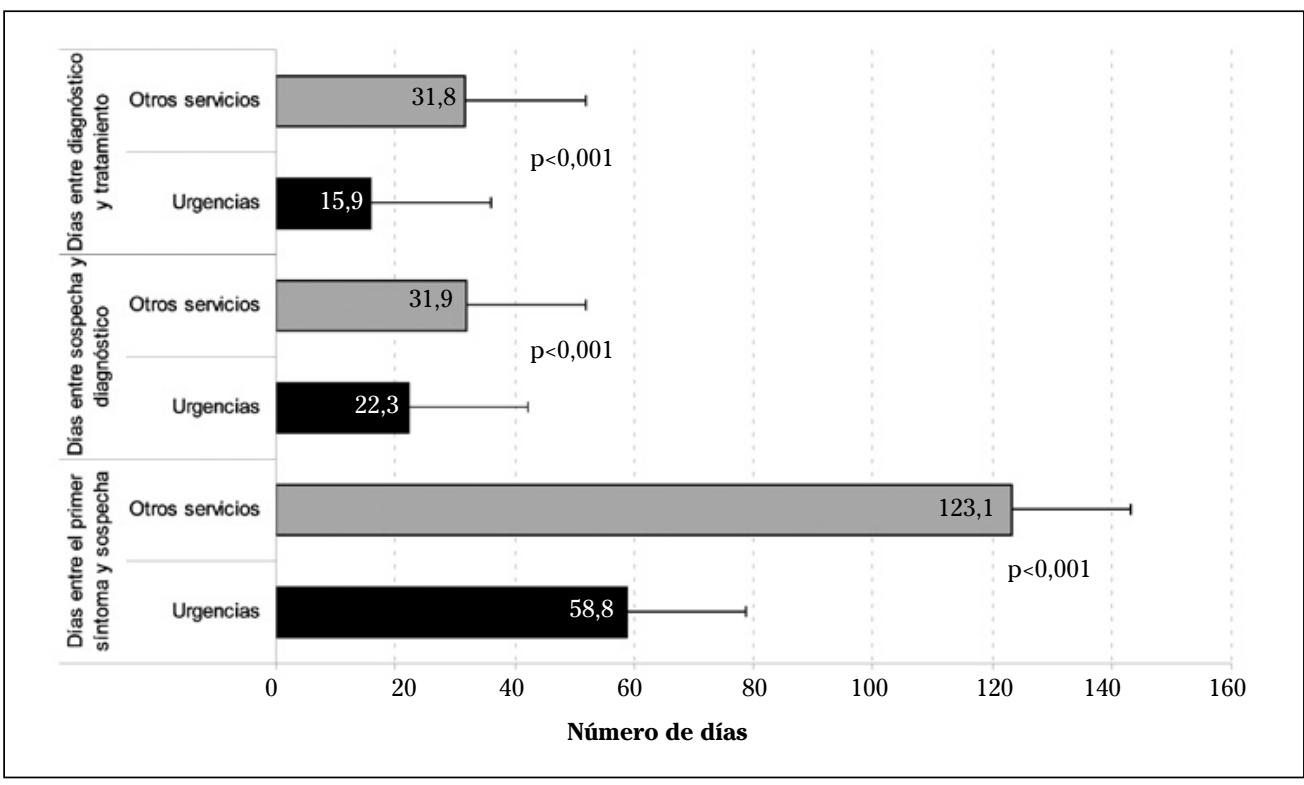

Figura 2. Días transcurridos entre (de abajo a arriba): el primer síntoma y el DS, el DS y la confirmación diagnóstica y el diagnóstico definitivo y el inicio del tratamiento; según el servicio de sospecha.

\section{DISCUSIÓN}

Existen pocos estudios y ninguno realizado en nuestro país sobre la importancia de los SUH en el diagnóstico de sospecha de la patología tumoral. En este trabajo se demuestra que los DS de cáncer son frecuentes en los SUH, más incluso que en servicios relacionados específicamente con el diagnóstico. Nuestros resultados parecen evidenciar que los profesionales de los SUH son muy capaces de detectar los síntomas y signos de cáncer pues sus DS superan a los de otros servicios en teoría más propicios para recibir casos nuevos de cáncer.

El cáncer debería ser detectado durante los exámenes periódicos de salud, ya sea a través del cribado poblacional o por el reconocimiento temprano de los signos y síntomas en $\mathrm{AP}^{11}$. Algunos estudios confirman que las sospechas diagnósticas en AP son muy habituales ${ }^{9}$, sin embargo, en nuestro estudio, la frecuencia de DS en los SUH superaron a los de AP, quizá porque es habitual derivar pacientes de AP a los SUH con una descripción semiológica, pero sin especificar la sospecha diagnóstica del profesional médico. Otro aspecto que también influye es la accesibilidad que tienen los SUH y su importante dotación tecnológica.

El porcentaje de casos que fueron sospechados en los SUH es inferior al $24 \%$ del Reino Unido ${ }^{18}$, pero habida cuenta del aumento progresivo de DS que se observa en 
nuestra serie no sería extraño un acercamiento, en el corto plazo, a esas cifras. La evolución del porcentaje de DS posterior al 2008 es llamativa y podría explicarse, bien porque el aumento de la incidencia de cáncer conlleva un incremento de la demanda de atención urgente, bien por la mejora creciente de la capacidad de los profesionales del SUH para detectar los síntomas vinculados a la existencia de un tumor que debuta abruptamente o por mayor disponibilidad de medios diagnósticos en este servicio. El incremento del porcentaje de DS en los SUH podría ser un argumento más a favor de la creación de unidades específicas de diagnóstico rápido del cáncer, cuya eficiencia ha sido confirmada en España ${ }^{19}$. Por otro lado, también debería suscitar un debate más profundo sobre la necesidad de mejorar la coordinación entre la AP y los servicios diagnósticos hospitalarios, para evitar que los profesionales de AP se vean abocados a derivar a pacientes a los SUH si desean una adecuada gestión de los casos cuando sospechan una enfermedad tan grave.

En nuestra serie hubo más DS en varones. Hargarten y col $^{11}$ también encontraron sobrerrepresentación masculina y lo atribuyeron a mayor incidencia de cáncer entre los hombres. Además, como éste es un hecho epidemiológico conocido, quizá conlleve un estado de alerta superior en los profesionales de los SUH cuando demanda atención un hombre Por otro lado, los cánceres más frecuentes en los hombres quizá cursen con una sintomatología más alarmante y específica que en las mujeres y, por ende, de ellos se sigue una mayor frecuentación de los SUH. Esta es una explicación plausible si tenemos en cuenta que el cáncer de pulmón, un tumor predominantemente masculino ${ }^{20}$, ha sido el DS más frecuente en los SUH, mientras que algunos de los más frecuentes en mujeres, como el de mama y útero, son objeto de programas de diagnóstico precoz, lo que no les permite avanzar hasta generar una situación que demande atención urgente.

Profundizando en las localizaciones tumorales, los cánceres con DS más frecuentes en los SUH, comparados con el resto de servicios, son el de tráquea-pulmón y el de colon, seguidos del gástrico. Kmietowi$\mathrm{cz}^{18}$, en un estudio poblacional, encontró una distribución de localizaciones similar en el Reino Unido, aunque en diferente orden. Nuestros resultados también corroboran los de un estudio piloto realizado en Barcelona ${ }^{21}$. La mayor frecuencia de estas localizaciones podría expresar la mayor incidencia general, como sucede con el de pulmón y el colon. Específicamente, en el caso del cáncer de pulmón también puede ser debido a que sus principales manifestaciones son dolor torácico y disnea, que son los dos síntomas que motivan más consultas en los SUH. Además, su sospecha diagnóstica se realiza mediante radiografía, que es una prueba accesible y barata y que se encuentra dentro de la mayoría de los protocolos diagnósticos en los SUH. Sería deseable implantar medidas de prevención primaria de ambos tumores en $\mathrm{AP}$ (programas de prevención del tabaquismo y de promoción de dieta saludable), puesto que la elevada frecuencia de DS en SUH detectada en nuestro estudio nos permite afirmar que tales tumores debutan con sintomatología subsidiaria de atención urgente, posiblemente por su estadio avanzado $\mathrm{y}$, por tanto, con mal pronóstico. En otros estudios, la frecuencia es todavía mayor y alrededor de 1 de cada 3 casos de cáncer de colon y de pulmón son presuntivamente diagnosticados en los $\mathrm{SUH}^{22,23}$. Por estas razones, incorporar a la rutina de la atención de las urgencias algoritmos que permitan diagnosticar presuntivamente estos cánceres podría ser beneficioso para los pacientes y el sistema. Por otro lado, un estudio realizado en dos servicios de AP de Mallor$\mathrm{ca}^{10}$, concluyó que el médico de AP puede mejorar su intervención en estos dos tumores (pulmón y colon). La mayor frecuencia de DS de cáncer de mama y próstata en servicios de atención no urgente se debería tanto a los programas de detección precoz como a que estos tumores no acostumbran a debutar con síntomas que requieran atención urgente.

Los hallazgos relacionados con el estadio y las metástasis son lógicos y consistentes con los resultados de otros estudios ${ }^{11}$. 
Hubo tumores más avanzados y más frecuencia de metástasis entre los sospechados en el SUH. Seguramente estos tumores se desarrollaron con sintomatología inespecífica hasta que el paciente o su médico de AP detectaron una situación abrupta que originó la consulta al SUH. También es coherente que hubiera menos DS de segundos primarios en los SUH. El tiempo medio transcurrido entre el primer y el segundo cáncer suele ser inferior a los 5 años ${ }^{24}$, por lo que los pacientes son diagnosticados del segundo tumor mientras todavía están en fase de seguimiento del primero, probablemente durante las pruebas de rutina en el servicio responsable del seguimiento del paciente.

Todos los tiempos estudiados son significativamente menores en el paciente atendido en el SUH que en el resto de servicios. Si el paciente debuta con síntomas que requieren atención urgente, se agilizan las pruebas diagnósticas, la derivación o el ingreso en el servicio especializado y el inicio del tratamiento. El tiempo transcurrido entre el primer síntoma y el DS es el que muestra mayor diferencia significativa entre el SUH (más corto) y el resto de servicios. Estos resultados coinciden con los de otros estudios ${ }^{10,25}$, en los que también se observa que el mayor tiempo de demora es el comprendido entre el primer síntoma y el DS. El estudio mallorquín ${ }^{10}$ encontró que el diagnóstico se demora más de un mes si lo realiza AP, excepto en los casos de cáncer de pulmón, próstata y colon, en los que el diagnóstico presuntivo es más precoz. En Asturias, dos de estos tumores son los que tienen DS más frecuente en los SUH, lo que sugiere que los servicios de AP no los detectan suficientemente y que deberían estar más alerta ante síntomas compatibles con la existencia de un tumor, lo que redundaría en un manejo de la enfermedad en un estadio de mejor pronóstico. La extensión tumoral -salvo en el cáncer de mama- no depende del tiempo transcurrido entre el primer síntoma y el diagnóstico ${ }^{10,21}$, pero este tiempo sí se correlaciona con el estadio y la supervivencia.

Nuestros resultados están limitados por un sesgo de información, si los datos del RHT no tuviesen la calidad necesaria. La calidad de la información de un registro de tumores que es fusión de otros varios con datos parciales podría adolecer de imprecisiones, redundancias o carencias, sobre todo durante los primeros años de la fusión. Para mitigar dicho sesgo, desechamos el primer bienio post-fusión (20042005) y analizamos los datos del septenio 2006-2012. Por otro lado, las conclusiones solo pueden referirse, sensu stricto, a la población asturiana, pero no es esperable que las peculiaridades regionales hicieran muy distintos los resultados de otras poblaciones españolas. Además, creemos que las recomendaciones para la gestión de los servicios que pueden derivarse de este estudio, serán igualmente válidas para otras regiones de España.

En conclusión, los SUH tienen gran importancia en el DS de algunos tumores, especialmente del sistema respiratorio (pulmón) y digestivo (colon), avanzados y con metástasis, sobre todo en hombres. Además, acortan todos los tiempos-algunos los reducen aproximadamente a la mitad-entre el primer síntoma y el DS, el de confirmación y el inicio del tratamiento.

\section{Agradecimientos}

Los autores agradecen al personal del Registro Hospitalario de Tumores del Principado de Asturias su ayuda en la gestión y depuración de la base de datos.

\section{BIBLIOGRAFÍA}

1. Ferlay J, Shin HR, Bray F, Forman D, Mathers C, PARKIN DM. GLOBOCAN 2008 v1.2, Cancer Incidence and Mortality Worldwide: IARC CancerBase No. 10 [base de datos en Internet]. Lyon: International Agency for Research on Cancer; 2010 [consultado 21-08-2013]. Disponible en: http://globocan.iarc.fr

2. LóPEZ-ABENTE G. Cancer surveillance in Spain: regional inequalities and peculiarities of temporal trends. Bull Cancer 2013;100: 11-14.

3. SÁnchez MJ, Payer T, De Angelis R, Larrañaga N, Capocaccia R, Martinez C; CIBERESP Working Group. Cancer incidence and mortality in Spain: estimates and projections for the pe- 
riod 1981-2012. Ann Oncol 2010; 21 Suppl 3: 30-36.

4. Bray F, Jemal A, Grey N, Ferlay J, Forman D. Global cancer transitions according to the Human Development Index (2008-2030): a population-based study. Lancet Oncol 2012; 13: 790-801.

5. Instituto Nacional de Estadística [base de datos en Internet]. Defunciones según causa de muerte 2010. Madrid: Instituto $\mathrm{Na}$ cional de Estadística; 2012 [consultado 2208-2013]. Disponible en: http://www.ine.es/ jaxi $/$ menu.do?type $=$ pcaxis \&path $=\% 2 F t 15 /$ p417\&file $=$ inebase \&L=0

6. Fernández N, Álvarez-Martín E, Morant-Ginestar C, Gènova-Maleras R, Gil A, Pérez-Gómez B et al. Burden of disease due to cancer in Spain. BMC Public Health 2009; 9: 42.

7. Fortún MT, Encina Y, Etxebarria MJ, Escudero JM, Bardón AI, Martínez S. Enfoque global del enfermo oncológico en urgencias. Datos epidemiológicos. An Sist Sanit Navar 2004; 27 Suppl 3: 9-16.

8. Underwood JM, Townsend JS, Stewart SL, BuChannan N, EkwUEme DU, Hawkins NA et al. Surveillance of Demographic Characteristics and Health Behaviors Among Adult Cancer Survivors - Behavioral Risk Factor Surveillance System, United States, 2009. MMWR 2012; 61: 1-23.

9. Simó-Cruzet E, Ureña-Tapia MM, Vernet-Vernet M, Sender-Palacios MJ, Larrosa-Sáez P, JovellFERNÁNDEZ E. Intervención del médico de familia en el diagnóstico del cáncer. Aten Primaria 2000; 26: 104-106.

10. Ruiz-Torrejón A, Ramos-Monserrat M, LloberaCánaves J. El médico de atención primaria y el diagnóstico de los pacientes con cáncer. Aten Primaria 2006; 37: 16-21.

11. Hargarten SW, Richards MJ, Anderson AJ. Cancer presentation in the emergency department: a failure of primary care. Am J Emerg Med 1992; 10: 290-293.

12. Vandyk AD, Harrison MB, Macartney G, RossWhite A, Stacey D. Emergency department visits for symptoms experienced by oncology patients: a systematic review. Support Care Cancer 2012; 20: 1589-1599.

13. Quiñones Pérez A, Álvarez Jiménez P, García SánCHEz MJ, FERrer FríAs MM. Furosemida nebulizada como tratamiento en urgencias de la disnea en pacientes con cáncer terminal. Emergencias 2011; 23: 208-210.

14. Gómez-Zorrilla Martín S, Riera-Mestre A, Leiva Pedraza D, Jordán Lucas S, Jacob Rodríguez J, Pujol Farriols R. Diagnóstico alternativo al tromboembolismo pulmonar en urgencias en pacientes oncológicos y no oncológicos. Emergencias 2013; 25: 92-98.

15. Ferrer ai, Vidal MJ, Tobeña M, Serrano S, RuizEcharri M, Millastre E. Evaluación sintomática del paciente oncológico en urgencias. Emergencias 2009; 21: 186-188.

16. Matilla A, González A, Castaño A, Colina F, Ramírez I, SANZ JM. Manual de Procedimientos en los Registros Hospitalarios de Tumores. Málaga: Sociedad Española de Anatomía Patológica; 2007.

17. April F, Constance P, Andrew J, Kanagaratnam S, Leslie S, MAX P et al, editors. International Classification of Diseases for Oncology: ICD$0.3^{\mathrm{a}}$ ed. Geneva: World Health Organization; 2000.

18. Кміетоwicz Z. One in three cases of cancer in patients over 70 are diagnosed at emergency admission. BMJ 2012; 345: e6402.

19. Bosch X, Moreno P, Ríos M, Jordán A, LóPEz-Soto A. Comparison of quick diagnosis units and conventional hospitalization for the diagnosis of cancer in Spain: a descriptive cohort study. Oncology 2012; 83: 283-291.

20. Shugarman LR, Mack K, Sorbero ME, Tian H, Jain AK, AsHwood JS et al. Race and sex differences in the receipt of timely and appropriate lung cancer treatment. Med Care 2009; 47: 774-781.

21. Casamituana M, Pozuelo A, López M, Segura JM, PERIS M [Internet]. Evaluación de un programa piloto de diagnóstico rápido de cáncer entre seis áreas básicas y su hospital de referencia. [consultado 05-08-2013]. Disponible en: http://www.postermedic.com/ parcdesalutmar/npimas062099/pdfbaja/npimas062099.pdf

22. Chen YL, Chang WC, Hsu HH, Hsu CW, Lin YY, Tsai SH. An evolutionary role of the ED: outcomes of patients with colorectal cancers presenting to the ED were not compromised. Am J Emerg Med 2013; 31: 646-650.

23. Beatty S, Stevens W, Stevens G, Kolbe J, Cox B. Lung cancer patients in New Zealand initially present to secondary care through the emergency department rather than by referral to a respiratory specialist. N Z Med J 2009; 122 : 33-41.

24. Lana-Pérez A, Folgueras-Sánchez MV, Díaz-Rodríguez S, del Olivo-del Valle Gómez M, CuetoEspinar A, López-GonzÁlez ML. Análisis de la supervivencia en pacientes con cáncer múltiple, Asturias, 1975-2004. Rev Esp Salud Publica 2008; 82: 167-177.

25. Bernal-Pérez M, Gómez-Bernal FJ, Gómez-Bernal GJ. Tiempos de demora en el diagnóstico del cáncer. Aten Primaria 2001; 27: 79-85. 
